\title{
Analysis of the Large- $n$ Limit of Multiple Orthogonal Polynomials and Non-intersecting Brownian Motions
}

\author{
L. Martínez Alonso* and E. Medina ${ }^{\dagger}$ \\ ${ }^{*}$ Departamento de Física Teórica II, Universidad Complutense. E28040 Madrid, Spain \\ ${ }^{\dagger}$ Departamento de Matemáticas, Universidad de Cádiz. E11510 Puerto Real, Cádiz, Spain
}

\begin{abstract}
In this work we use Riemann-Hilbert problems for multiple orthogonal polynomials in order to derive string equations associated to Lax-Orlov pairs operators. These string equations provide us with a useful tool to analyze the large $n$-limit of the related hierarchies. The results are finally applied to the study of the associated random matrix models (Gaussian Hermitian matrix models with an external source) and non-intersecting Brownian motions starting from a fix point.
\end{abstract}

Keywords: Riemann-Hilbert problem, Multiple orthogonal polynomials, Random matrix models PACS: $02.30 \mathrm{Ik}$

\section{INTRODUCTION}

It is well known that the set of orthogonal polynomials $P_{n}(x)=x^{n}+\cdots$, with respect to an exponential weight

$$
\int_{-\infty}^{\infty} P_{n}(x) P_{m}(x) e^{V(\mathbf{c}, x)} d x=h_{n} \delta_{n m}, \quad V(\mathbf{c}, x):=\sum_{k \geq 1} c_{k} x^{k}
$$

is an essential ingredient of the methods [1]-[2] for studying the large- $n$ limit of the Hermitian matrix model

$$
Z_{n}=\int d M \exp (\operatorname{Tr} V(\mathbf{c}, M))
$$

These polynomials satisfy a pair of string equations that can be formulated in terms of a pair of Lax-Orlov operators. In particular, one of these relations is the standard three-term relation for orthogonal polynomials.

More recently, it has been found [3]-[4] that multiple orthogonal polynomials are connected to the Gaussian Hermitian matrix model with an external source term $A M$, where $A$ is a fixed diagonal $n \times n$ real matrix. The partition function of this model is given by

$$
Z_{n}=\int d M \exp \left(-\operatorname{Tr}\left(\frac{1}{2} M^{2}-A M\right)\right)
$$

It turns out that if the eigenvalues of $A$ are given by $a_{j},(j=1, \ldots, q)$ with multiplicities $n_{j}$, then the expectation values $P(\mathbf{n}, z)=\mathbb{E}[\operatorname{det}(z-M)], \mathbf{n}:=\left(n_{1}, \ldots, n_{q}\right)$, are multiple orthogonal polynomials with respect to the Gaussian weights

$$
w_{j}(x)=\exp \left(a_{j} x-\frac{1}{2} x^{2}\right), \quad j=1,2, \ldots, q .
$$

These matrix models are deeply connected to one-dimensional non-intersecting Brownian motion [5].

The Riemann-Hilbert (RH) problem of Fokas-Its-Kitaev for orthogonal polynomials was generalized by Van Assche, Geronimo and Kuijlaars [6] to characterize multiple orthogonal polynomials. Our starting point is this RH problem, and more specifically, its fundamental solution $f$, which is a $n \times n$ matrix such that the multiple orthogonal polynomials are elements in the first row. By applying standard methods in the theory of multi-component integrable systems [7] we formulate string equations for the elements of the first row of the matrix function $f$.

The other fundamental ingredient is due to Takasaki and Takebe [8] who showed that the dispersionless limit of a row of a matrix-valued KP wave function is a solution of the zero genus Whitham hierarchy. This result suggests the use of Lax-Orlov operators in order to characterize the large-n limit in terms of quasiclassical (dispersionless limit) expansions. Thus, we show that the leading term of the expansion of the first row of $f$ is determined by a system of dispersionless string equations for $q+1$ Lax-Orlov functions $\left(z_{\alpha}, m_{\alpha}\right), \alpha=0,1, \ldots, q$, in the phase space of the Whitham hierarchy. Finally, we illustrate the applications of our results to models of random matrix ensembles and non-intersecting Brownian motions. CP1168, Vol. 1, Numerical Analysis and Applied Mathematics, International Conference 2009
edited by T. E. Simos, G. Psihoyios, and Ch. Tsitouras 02009 American Institute of Physics 978-0-7354-0705-3/09/\$25.00 


\section{RIEMANN-HILBERT PROBLEMS AND MULTIPLE ORTHOGONAL POLYNOMIALS}

Given a matrix function $g=g(z)(z \in \mathbb{R})$ such that $\operatorname{det} g(z) \equiv 1$, we will consider the RH problem

$$
m_{-}(z) g(z)=m_{+}(z), \quad z \in \mathbb{R}
$$

where $m(z)$ is a matrix holomorphic function in $\mathbb{C}-\mathbb{R}$ and $m_{ \pm}(z):=\lim _{\mathcal{E} \rightarrow 0^{+}} m(z \pm i \varepsilon)$. We are interested in solutions $f=f(\mathbf{s}, z)$ of (4) depending on $q$ discrete variables $\mathbf{s}=\left(s_{1}, \ldots, s_{q}\right) \in \mathbb{Z}^{q}$ such that

$$
f(\mathbf{s}, z)=\left(I+\mathscr{O}\left(\frac{1}{z}\right)\right)\left(\sum_{\alpha=0}^{q} z^{s \alpha} E_{\alpha}\right), \quad z \rightarrow \infty, \quad \text { where } \quad s_{0}:=-\sum_{i=1}^{q} s_{i} .
$$

Here, we are denoting by $E_{\alpha \beta}$ the matrices $\left(E_{\alpha \beta}\right)_{\alpha^{\prime} \beta^{\prime}}=\delta_{\alpha \alpha^{\prime}} \delta_{\beta \beta^{\prime}}$ of the canonical basis and, in particular, its diagonal members are denoted by $E_{\alpha}:=E_{\alpha \alpha}$.

The set of points $\mathbf{s} \in \mathbb{Z}^{q}$ for which (4) admits a unique solution $f(\mathbf{s}, z)$ satisfying (5) will be denoted by $\Gamma$. The solution $f(\mathbf{s}, z)$ will be referred to as the fundamental solution of the RH problem (4).

In order to introduce the multiple orthogonal polynomials of type I we start with $q$ exponential weights $w_{i}$ on the real line $w_{i}(x):=e^{-V\left(\mathbf{c}_{i}, x\right)}, \mathbf{c}_{i}=\left(c_{i 1}, c_{i 2}, \ldots\right) \in \mathbb{C}^{\infty}$, where $V(\mathbf{c}, x)$ is defined as in (1) and we are assuming that only a finite number of coefficients $c_{n}$ are different from zero. We take $\mathbf{n}=\left(n_{1}, \ldots, n_{q}\right) \in \mathbb{N} q$, with $|\mathbf{n}|:=\sum_{j=1}^{q} n_{j} \geq 1$. Then if $\mathbf{A}(\mathbf{n}, x)=\left(A_{1}(\mathbf{n}, x), \ldots, A_{q}(\mathbf{n}, x)\right)$ are polynomials such that

$$
A_{j}(\mathbf{n}, x) \quad \text { has degree } n_{j}-1 \quad \text { for } \quad n_{j} \geq 1 \quad \text { and } \quad A_{j}(\mathbf{n}, z) \equiv 0 \text { for } n_{j}=0,
$$

which satisfy the orthogonality relations

$$
\int_{\mathbb{R}} \frac{d x}{2 \pi i} x^{l}\left(\sum_{j=1}^{q} A_{j}(\mathbf{n}, x) w_{j}(x)\right)= \begin{cases}0 & l=0,1, \ldots,|\mathbf{n}|-2, \\ 1 & l=|\mathbf{n}|-1,\end{cases}
$$

then $A_{j}(\mathbf{n}, x)$ are called multiple orthogonal polynomials of type $\mathbf{I}$. We assume that the solution $\mathbf{A}(\mathbf{n}, x)$ of $(6)$ and (7) is unique for each $\mathbf{n}$ such that $|\mathbf{n}| \geq 1$.

On the other hand, we consider $q$ exponential weights $w_{i}$ on the real line $w_{i}(x):=e^{V\left(\mathfrak{c}_{i}, x\right)}$. Given $\mathbf{n}=\left(n_{1}, \ldots, n_{q}\right) \in$ $\mathbb{N}^{q}$, if $P(\mathbf{n}, x)=x^{|\mathbf{n}|}+\cdots$ is a monic polynomial satisfying

$$
\int_{\mathbb{R}} P(\mathbf{n}, x) w_{i}(x) x^{j} d x=0, \quad j=0, \ldots, n_{i}-1, \quad i=1, \ldots, q,
$$

then $P(\mathbf{n}, x)$ is called a multiple orthogonal polynomial of type II. Again we assume that the solution $P(\mathbf{n}, x)$ of $(8)$ is unique for each $\mathbf{n} \in \mathbb{N}^{q}$.

The RH problem which characterizes these polynomials [5],[6] are determined by

$$
g_{I}(z)=\left(\begin{array}{cc}
1 & \mathbf{0}^{t} \\
-\mathbf{w}(z) & I_{q}
\end{array}\right), \quad g_{I I}(z)=\left(\begin{array}{cc}
1 & \mathbf{w}(z)^{t} \\
\mathbf{0} & I_{q}
\end{array}\right)
$$

for type I and type II orthogonal polynomials respectively. Here $\mathbf{0}$ stands by the vector of $\mathbb{C}^{q}$ with all its components equal to zero, $I_{q}$ for the identity matrix of dimension $q$ and we are denoting $\mathbf{w}(z)=\left(w_{1}(z), \ldots, w_{q}(z)\right)^{t}$.

\section{STRING EQUATIONS AND LAX-ORLOV OPERATORS}

We introduce the $q$ pairs of shift operators $T_{i}, T_{i}^{*}$ acting on functions $h(\mathbf{s})(\mathbf{s} \in \Gamma)$ defined as

$$
\left(T_{i} h\right)(\mathbf{s}):=\left\{\begin{array}{ccc}
h\left(\mathbf{s}-\mathbf{e}_{i}\right) & \text { if } & \mathbf{s}-\mathbf{e}_{i} \in \Gamma, \\
0 & \text { if } & \mathbf{s}-\mathbf{e}_{i} \notin \Gamma,
\end{array} \quad\left(T_{i}^{*} h\right)(\mathbf{s}):=\left\{\begin{array}{ccc}
h\left(\mathbf{s}+\mathbf{e}_{i}\right) & \text { if } & \mathbf{s}+\mathbf{e}_{i} \in \Gamma, \\
0 & \text { if } & \mathbf{s}+\mathbf{e}_{i} \notin \Gamma,
\end{array}\right.\right.
$$

where $\mathbf{e}_{i}$ are the elements of the canonical basis of $\mathbb{C}^{q}$, and $\Gamma$ stands for the domain in which the RH problem has a unique solution. In terms of these operators and using standard techniques in theory of integrable systems we obtain: 
First system of string equations

The first row of the fundamental solution $f$ satisfies the equations

$$
z\left(E_{0} f\right)(\mathbf{s}, z)=\left(T_{i}+u_{i}(\mathbf{s})+\sum_{j} v_{j}(\mathbf{s}) T_{j}^{*}\right)\left(E_{0} f\right)(\mathbf{s}, z),
$$

with $\mathbf{s}$ in appropriate domains and $u_{i}(\mathbf{s}), v_{i}(\mathbf{s})$ certain scalar functions.

\section{Lax operators}

The functions $f_{0 i}(\mathbf{s}, z), i=1,2, \ldots, q$ can be written in terms of some dressing operators as $f_{0 i}(\mathbf{s}, z)=\left(G_{i} \xi_{i}\right)(\mathbf{s}, z)$, $\xi_{i}(\mathbf{s}, z):=z^{S_{i}-1}$, with $\mathbf{s}$ in some appropriate domains and $G_{i}$ are dressing operators that can be expanded as

$$
G_{i}=\sum_{n \geq 1} \alpha_{i n}(\mathbf{s}) T_{i}^{n-1},
$$

being the coefficients $\alpha_{i n}(\mathbf{s})$ matrix functions. In terms of the dressing operators we define the Lax operators as $\mathscr{Z}_{i}:=G_{i} T_{i}^{*} G_{i}^{-1}=\gamma_{i}(\mathbf{s}) T_{i}^{*}+\sum_{n \geq 0} \gamma_{i n}(\mathbf{s}) T_{i}^{n}$. Then, the functions $f_{0 i}$ satisfy the equations

$$
z f_{0 i}(\mathbf{s}, z)=\left(\mathscr{Z}_{i} f_{0 i}\right)(\mathbf{s}, z)
$$

\section{Second system of string equations}

The function $f$ satisfies the equation

$$
\left(D_{z}+\mathscr{H}\right)\left(E_{0} f\right)(\mathbf{s}, z)=0
$$

with $\mathbf{s}$ in a certain domain and where $D_{z}$ is a covariant derivative and $\mathscr{H}$ a difference operator that can be expressed in terms of the shift operators $T_{i}^{*}, i=1, \ldots, q$.

Orlov operators

The Orlov operators $\mathscr{M}_{i}$ can be defined by $\mathscr{M}_{i}:=G_{i} \cdot s_{i} \cdot T_{i} \cdot G_{i}^{-1}=\sum_{n \geq 1} \mu_{i n}(\mathbf{s}) T_{i}^{n}$. They satisfy $\left[\mathscr{Z}_{i}, \mathscr{M}_{i}\right]=\mathbb{I}$ and in terms of these operators we have that the functions $f_{0 i}$ satisfy the equations

$$
\partial_{z} f_{0 i}(\mathbf{s}, z)=\left(\mathscr{M}_{i} f_{0 i}\right)(\mathbf{s}, z)
$$

\section{THE LARGE $n$ LIMIT AND APPLICATIONS}

In order to define the large- $n$ limit we consider the fundamental solution of the RH problems for large values of the discrete parameters $s_{i}$, more specifically $s_{i}>>1$, in the type I case and $s_{i}<<-1$, in the type II case. We introduce slow variables $t_{i}:=\varepsilon s_{i}$ (with $\varepsilon$ being a small parameter) and rescale the exponent in the weight functions as

$w_{i}(\varepsilon, z)=\exp \left(\mp \frac{V\left(\mathbf{c}_{i}, z\right)}{\varepsilon}\right)$, where the exponent sign is negative (positive) for polynomials of type I (type II). Then, we take the continuum limit $\varepsilon \rightarrow 0$ in which the discrete parameters $s_{i}$ tends to $+\infty(-\infty)$ and the slow variables $t_{i}$ become continuous variables.

The shift operators, dressing operators and Lax-Orlov operators can be expressed in terms of the continuous variables $\mathbf{t}$ and the small parameter $\varepsilon$. Now, making use of a particular ansatz for the fundamental solution we can introduce a set of $q+1$ functions $z_{\alpha}(\mathbf{t}, p), m_{\alpha}(\mathbf{t}, p), \alpha=0,1, \ldots, q$, depending on the continuos variables $\mathbf{t}$ and a complex variable $p$. These functions provide the classical limit of the Lax-Orlov operator i.e.

$$
\left(\mathscr{Z}_{\alpha} f_{0 \alpha}\right)\left(\mathbf{t}, z_{\alpha}(\mathbf{t}, p)\right)=z_{\alpha}(\mathbf{t}, p) f_{0 \alpha}\left(\mathbf{t}, z_{\alpha}(\mathbf{t}, p)\right), \quad\left(\mathscr{M}_{\alpha} f_{0 \alpha}\right)\left(\mathbf{t}, z_{\alpha}(\mathbf{t}, p)\right)=\left(m_{\alpha}+\mathscr{O}(\varepsilon)\right) f_{0 \alpha}\left(\mathbf{t}, z_{\alpha}(\mathbf{t}, p)\right),
$$

and can be expanded as

$$
\begin{aligned}
& z_{0}=p+\sum_{n=1}^{\infty} \frac{v_{0 n}(\mathbf{t})}{p^{n}}, \quad p \rightarrow \infty, \quad z_{i}=\frac{v_{i}(\mathbf{t})}{p-u_{i}(\mathbf{t})}+\sum_{n=0}^{\infty} v_{i n}(\mathbf{t})\left(p-u_{i}(\mathbf{t})\right)^{n}, \quad p \rightarrow u_{i}(\mathbf{t}), \quad i=1, \ldots, q \\
& m_{\alpha}=\frac{t_{\alpha}}{z_{\alpha}}-\sum_{n \geq 1} \frac{n S_{\alpha n}(\mathbf{t})}{z_{\alpha}^{n+1}}, \quad \text { as } z_{\alpha} \rightarrow \infty, \quad \alpha=0,1, \ldots, q .
\end{aligned}
$$



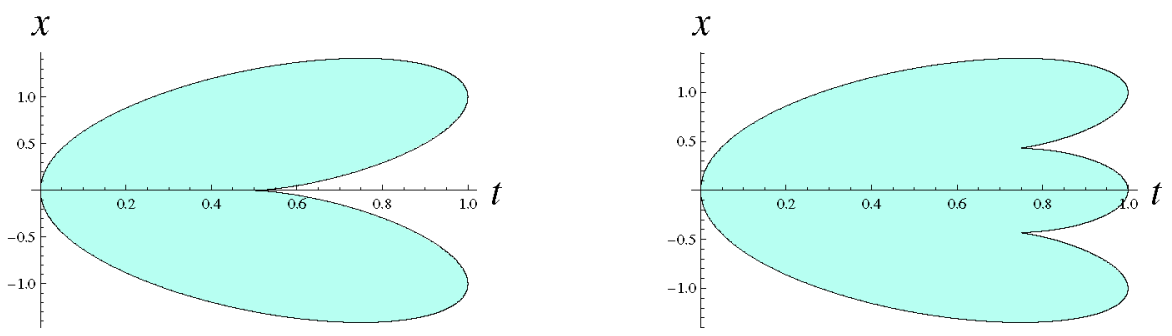

FIGURE 1. Limit support for Brownian motions with two and three end points

Taking into account (11) we have that the limit $\varepsilon \rightarrow 0$ of (9)-(10) is the system of string equations

$$
z_{0}=z_{1}=\cdots=z_{q}=E(p), \quad m_{0}=m_{1}-V^{\prime}\left(\mathbf{c}_{1}, z_{1}\right)=\cdots=m_{q}-V^{\prime}\left(\mathbf{c}_{q}, z_{q}\right)=-H(p),
$$

where $E$ and $H$ are certain rational functions of $p$ with a set of coefficients $u_{i}(\mathbf{t}), v_{i}(\mathbf{t}), i=1, \ldots, q$. These coefficients can be obtained by solving the system of hodograph equations (12).

If we assume that the roots of the multiple orthogonal polynomials are distributed as $n \rightarrow \infty$ with a continuos density on some compact (possibly disconnected) support $I=\bigcup_{k=1}^{d} I_{k} \subset \mathbb{R}$, we can deduce that the Orlov functions are the Cauchy transforms of the root densities, and the end points of the segments $I_{k}$ are the branch points of the functions $p_{\alpha}(\mathbf{t}, z)$, inverse functions of $z_{\alpha}(\mathbf{t}, p)$. Thus, the system of string equations (12) allows us to determine these points.

Moreover, the Gaussian matrix models with an external source (2) are related to the multiple orthogonal polynomials respect to the Gaussian weights (3), [3]-[4]. In this case the quasiclassical limit is characterized by $\varepsilon=1 / n$ and $t_{0}=1$, and the Orlov functions are related to the joint probability density for the eigenvalues of $M$.

Besides, these models are also connected to one-dimensional nonintersecting Brownian motions. Indeed, we have that the probability density for the eigenvalues $\left(\lambda_{1}, \ldots, \lambda_{n}\right)$ is the same to the probability density at time $t \in(0,1)$ for the positions $\left(x_{1}, \ldots, x_{n}\right)$ of nonintersecting Brownian motions starting at the origin at $t=0$ and ending at $q$ fixed points $b_{i}, i=1, \ldots, q$ at $t=1$. The relation between the parameters of both models is given by $x_{j}=\lambda_{j} \sqrt{t(1-t)}$, $a_{k}=b_{k} \sqrt{t(1-t)^{-1}}$.

As an example we illustrate in Figure 1 the cases $q=2$ with ending points at the symmetric positions \pm 1 , and $q=3$ with the ending points at 0 and \pm 1 . In the last case numerical analysis is used to determine the branch points.

\section{CONCLUSIONS}

The non-intersecting $n$-Brownian motions with 1 starting point and $q \geq 2$ ending points in the large $n$-limit is connected with the Gaussian Hermitian matrix model with an external source. Besides, the distribution of the eigenvalues for these models can be analyzed in terms of multiple ortogonal polynomials.

Making use of these relations and applying methods in theory of integrable systems we have developed a scheme for determining the limit support for the Brownian motions for arbitrary $q$. The extensions of these methods to nonintersecting Brownian motions with $q_{1} \geq 2$ starting points and $q_{2} \geq 2$ ending points remains an open problem.

\section{REFERENCES}

1. P. Di Francesco, P. Ginsparg and Z. Zinn-Justin, Phys. Rept. 254, 1-2 (1995), 133 pp.

2. P. Deift, "Orthogonal Polynomials and Random Matrices: A Riemann-Hilbert approach" in Courant Lecture Notes in Mathematics, Vol. 3, Amer. Math. Soc., Providence R.I. 1999.

3. P.M. Bleher and A.B.J. Kuijlaars, Comm. Math. Phys. 252, (2004), pp. 43-76.

4. P.M. Bleher and A.B.J. Kuijlaars, , Comm. Math. Phys. 270, (2007), pp. 481-517.

5. E. Daems and A.B.J. Kuijlaars, J. of Approx. Theory 146, (2007), pp. 91-114.

6. W. Van Assche, J.S. Geronimo, and A.B.J. Kuijlaars, Riemann-Hilbert problems for multiple orthogonal polynomials, In: Special Functions 2000: Current Perspectives and Future Directions (J. Bustoz et al., eds.), Kluwer, Dordrecht, (2001), pp. $23-59$.

7. L. Martínez Alonso, E. Medina and M. Mañas, J. Math. Phys. 47, 83512 (2006) 22 pp.

8. K. Takasaki and T. Takebe, Physica D 235, (2007) pp. 109-125. 
Copyright of AIP Conference Proceedings is the property of American Institute of Physics and its content may not be copied or emailed to multiple sites or posted to a listserv without the copyright holder's express written permission. However, users may print, download, or email articles for individual use. 